\title{
Properties of sunspot penumbral grains observed with Hinode
}

\author{
Y. Zhang ${ }^{1,2}$ and K. Ichimoto ${ }^{1}$ \\ 1 Kwasan and Hida Observatories, Kyoto University, Yamashina-ku, 607-847 Kyoto, Japan \\ e-mail: [zhangyin;ichimoto] @kwasan.kyoto-u.ac.jp \\ 2 Key Laboratory of Solar Activity, National Astronomical Observatories, Chinese Academy of Sciences, Chaoyang District, \\ 100012 Beijing, PR China \\ e-mail: zhangyin@bao.ac.cn
}

Received 19 September 2011 / Accepted 9 July 2013

\begin{abstract}
Context. Penumbral grains (PGs) are small-scale (subarcsec) bright features found in the bright penumbral filament in the outer parts of sunspots.

Aims. We aim to study properties of PGs at the blue continuum (4504 $\AA$ ) and the G-band (4305 $\AA$ ) by using simultaneous seeing free data obtained by Broadband Filter Imager (BFI) on board Hinode.

Methods. We use an automatic identification and tracking algorithm to identify PGs, which were observed in a period of 170 min in the blue continuum and 88 min in the G-band.

Results. Our results indicate that 776 PGs were identified in the blue continuum, and 413 PGs were identified in the G-band,. A statistical study reveals that about 55\% of PGs move toward the umbra, about $13-19 \%$ of PGs move toward the surrounding granulation, and the rest are relatively static. The inward moving PGs are mostly located in the inner penumbra (up to 0.6 of the distance from the umbra to the photosphere) and outward moving PGs are located in the outer penumbra. In the blue continuum (and $\mathrm{G}$ band), the average lifetime, speed, and brightness of inward moving PGs are 14.7 (13.5) min, $0.71(0.70) \mathrm{km} \mathrm{s}^{-1}$ and 0.89 (0.88) of the quiet Sun. For outward moving PGs, the average lifetime, speed and brightness are 8.0 (7.0) min, $0.93(0.65) \mathrm{km} \mathrm{s}^{-1}$ and $0.99(0.98)$ of the quiet Sun. For PGs that are relatively static, the average lifetime and brightness are $7.6(6.0)$ min and $0.94(0.91)$ of the quiet Sun. Moreover, our observational results show that the correlation between the temporal evolutions of the speed and brightness is complex.
\end{abstract}

Key words. Sun: photosphere - sunspots

\section{Introduction}

Penumbral grains (PGs), which appear either isolated or as part of comet-like bright filaments that move toward the sunspot umbra, were discovered by Muller (1973a). Subsequently, a series of works have confirmed that PGs moved toward the umbra with proper motions of several hundreds meters per second and with a lifetime of several hours (Muller 1973b, 1976; Tönjes \& Wöhl 1981). Since then, rare observational studies have been done about this topic, because successful observations require an extended period of excellent seeing. Recently, Sobotka and his collaborators have researched a series with the sub-arcsecond and sub-minute resolution data (Sobotka et al. 1999a,b; Sobotka \& Sütterlin 2001). Using observations at $4680 \pm 50 \AA$ from the Swedish Vacuum Solar Telescope (SVST) in La Palma, they found that almost $75 \%$ of PGs move toward the umbra, and the rests move toward the surrounding granulation. The inward moving PGs dominate the inner penumbra and the outward moving PGs dominate the outer penumbra. For inward moving PGs, the typical speed of proper motion is $0.4 \mathrm{~km} \mathrm{~s}^{-1}$, and the median lifetime is $29 \mathrm{~min}$. For outward moving ones, the speed is $0.5 \mathrm{~km} \mathrm{~s}^{-1}$, and the median lifetime is $22 \mathrm{~min}$. Furthermore, using observations in the $\mathrm{G}$ band (4305 $\AA$ ) from Dutch Open Telescope in La Palma, they found that $54 \%$ of PGs move toward the umbra, and $46 \%$ move toward the surrounding granulation.
Most inward moving PGs locate in the inner penumbra. Their average lifetime and median speed are $50 \mathrm{~min}$ and $0.52 \mathrm{~km} \mathrm{~s}^{-1}$, respectively. Most outward moving PGs are observed in the outer penumbra and their average lifetime and median speed are $31 \mathrm{~min}$ and $0.75 \mathrm{~km} \mathrm{~s}^{-1}$, respectively. These results generally confirm their previous studies.

Many models have been proposed to explain the fine structure of penumbra and also the properties of PGs. The umcombed penumbral model (Solanki \& Montavon 1993) has suggested a nearly horizontal flux tube embedded in more vertical background fields. On the basis of this model, Schlichenmaier et al. (1998) proposed a moving flux tube model, in which a thin flux tube carrying hot Evershed flow rises into the background fields and migrates toward the umbra. According to the simulation, PGs are the source of the cross section of the magnetic flux tubes, along which hot sub-photospheric plasma flow upward with a few $\mathrm{km} \mathrm{s}^{-1}$. They migrate from the outer edge of the penumbra toward the umbra with a proper speed exceeding $2 \mathrm{~km} \mathrm{~s}^{-1}$. This mechanism was later found to be unable to explain the brightness of the penumbra (Solanki \& Rüedi 2003; Schlichenmaier \& Solanki 2003), but the moving flux tube model survived as a reasonably good, yet idealized, description of the penumbral magnetic field.

With the high spectral resolution data obtained by Hinode, PGs are recently observationally identified as the location of 

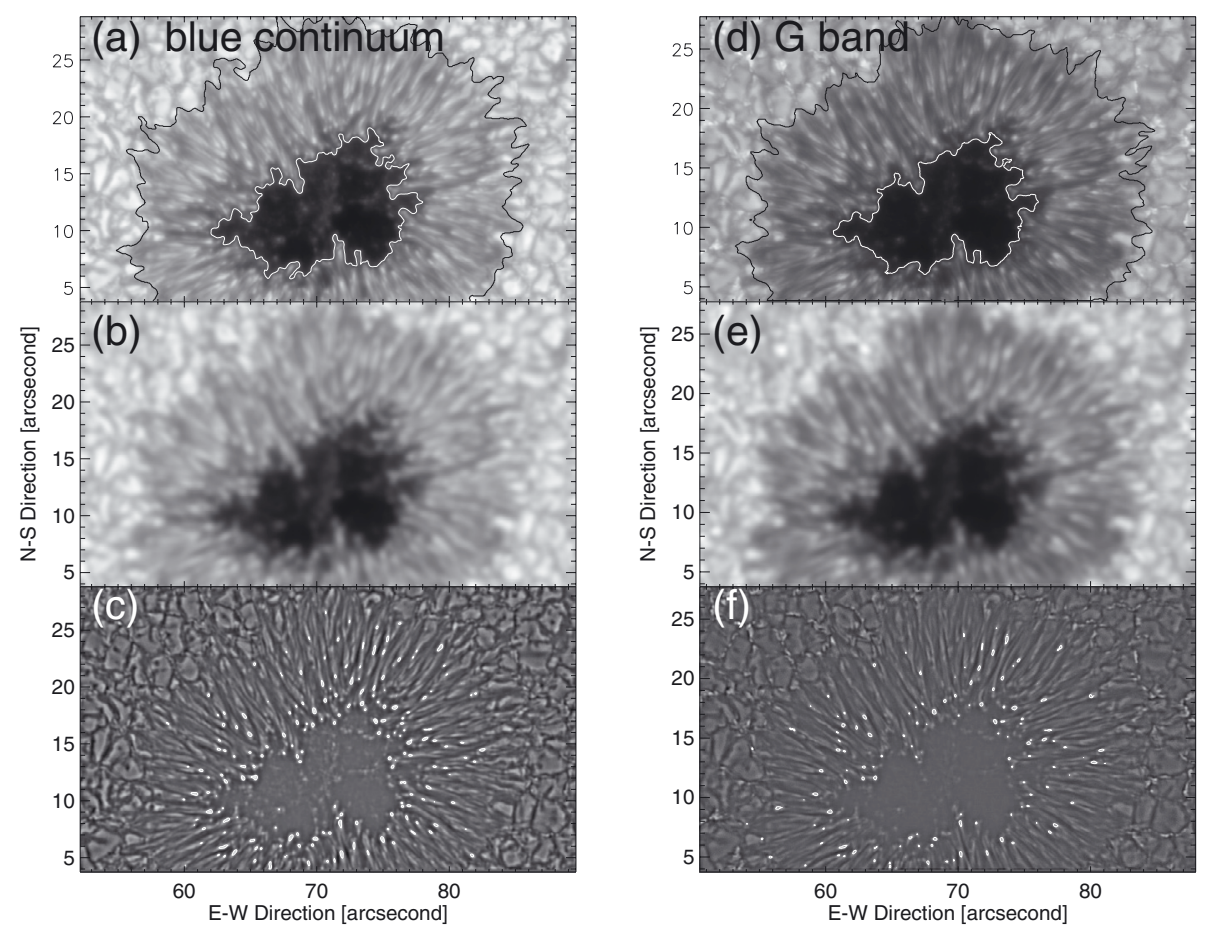

Fig. 1. Observed sunspot and penumbral grain (PG) identification. a) Filtergram in the blue continumm. b) Filtergram smoothed by a boxcar $(10 \times 10$ pixels $)$. c) Differential image a), b). The contours highlight PGs, which are identified by our detection procedure. Panels d) to f) show the same but for the G-band.

rising hot gas that serve as the sources of the Evershed flow (Ichimoto et al. 2007; Franz \& Schilchenmaier 2009). Sobotka \& Jurčák (2009) found that PGs appear in regions with a weaker and more horizontal magnetic field with stronger up-flows in the low photosphere. According to the rising flux tube model, the sources of Evershed flow should move inward, whereas the nature of PGs moving outward is not known. Meanwhile, studies based on local-correlation-tracking methods that use high spatial resolution data have revealed that all features in inner penumbra move inward, and all features in outer penumbra move outward (Deng et al. 2007; Min et al. 2009). However, the outward motions detected by this method may be due to the Evershed outflow, which take place in dark lanes in outer penumbra. Thus, careful sampling of only bright features to investigate the real motion of the bright grains is desirable.

A good observational understanding of the properties of PGs based on high spatial and temporal resolution photospheric filtergrams without the seeing effect will certainly help us to understand the nature of the penumbra. With the launch of the Hinode spacecraft (Kosugi et al. 2007), these data can be obtained from time to time. It provides more detail and fine structures of penumbra. Our purpose here is to study the properties of PGs by using seeing free data. The observations and data reduction are described in Sect. 2. In Sect. 3, we present the results and summarize and discuss them in Sect. 4.

\section{Observations and data reduction}

\subsection{Observations}

The Broadband Filter Imager (BFI) of the Solar Optical Telescope (Tsuneta et al. 2007) aboard Hinode produces photometric images in six bands ( $\mathrm{CN}$ band, $\mathrm{Ca}$ II H-line, G-band, and 3 continuum bands) at the highest spatial resolution and at rapid cadence (10-30 s typical). A mature $\alpha$ sunspot in NOAA 10944 located at S06W04 $(\mu=0.99)$ was observed by BFI on March 1, 2007 in the blue continuum (4504 $\AA$ ) and in the G-band (4305 $\AA$ ). The spatial and temporal resolution is about $0.2^{\prime \prime}$ and $7 \mathrm{~s}$, respectively. The FOV is $56^{\prime \prime} \times 28^{\prime \prime}$ with a pixel size of $0.054^{\prime \prime}$. For further analysis, we selected a $40^{\prime \prime} \times 24^{\prime \prime}(740 \times 440$ pixels $)$ field after alignment. The observational period in the blue continuum (and $G$ band) is from 00:14 UT to 03:04 UT (from 01:36 UT to 03:04 UT). We apply a subsonic $k-\omega$ filter with a cutoff speed of $7 \mathrm{~km} \mathrm{~s}^{-1}$ to remove the effects of the 5-min oscillation. Before further data reduction, we normalize intensity in each filtergram by the average intensity of the quiet Sun.

\subsection{Identification of $P G s$}

We show an example of the filtergrams of both wavelengths in Figs. 1a and d. The boundaries between surrounding granulation and penumbra and between penumbra and umbra are determined by assuming an intensity threshold relative to the quiet Sun of $0.9(0.9)$ and $0.4(0.3)$ for the blue continuum (and $\mathrm{G}$ band), which is indicated by black and white contour. It shows that the sunspot umbra is encircled by well developed penumbra, even though it is not a perfect circle and its limb is uneven. If we regard the umbra as an ellipse, its eccentricity is about 0.8 for both wavelengths. The nearest distance from the sunspot center to the boundary between umbra and penumbra is less than $4^{\prime \prime}$, while the farthest distance is more than $7^{\prime \prime}$. The average width of penumbra is about $8^{\prime \prime}$ for both wavelengths.

To isolate individual PGs, a simple image segmentation method based on an edge enhancement algorithm is used. This method was described in detail in Sobotka et al. (1997), so we just give a brief introduction here. Figure $1 \mathrm{~b}$ and $1 \mathrm{e}$ show smoothed images (boxcar $10 \times 10$ pixels) from the original ones. The differential image between Figs. 1a and b (Figs. 1d and e) is shown in Fig. 1c (1f). In Fig. 1c (1f), PGs were distinguishable by the image segmentation method. Then, we apply a binary mask on the differential image, setting pixel values higher than an empirically determined threshold ( 0.1 for the blue continuum and 0.14 for the $G$ band) to 1 and the rest to 0 . We bear in mind 

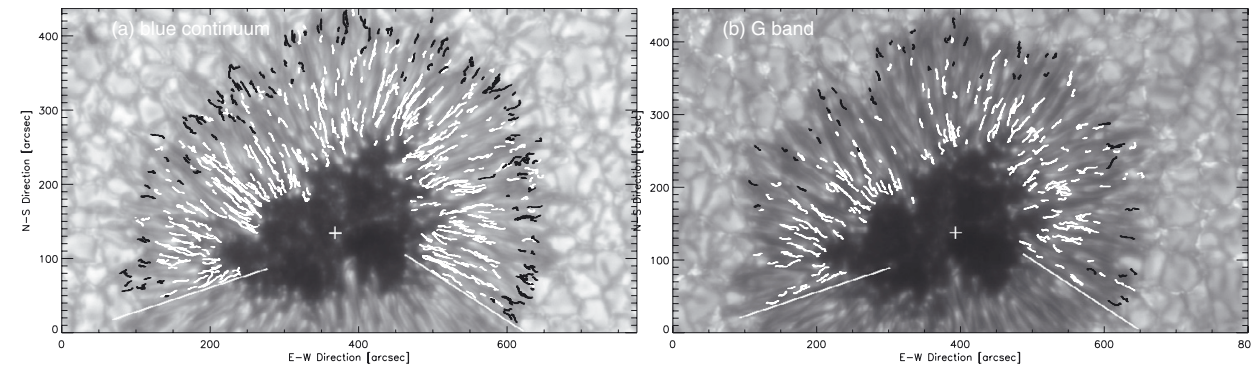

Fig. 2. Trajectories of INW (white) and OUT (black) PGs for blue continuum (panel a)) and G-band (panel b)). The underlying image is the filtergrams of each wavelength. that the pixel value in differential images has only local meaning. It measures the extent the brightness of one pixel is compared to the average brightness of its neighboring pixels. After that, we define another binary mask to exclude bright features in the quiet Sun and umbra. To create the mask, we average all filtergrams through the whole observation. We then set pixel values according to the following criteria: if the pixel value falls in the range of 0.35 and 0.9 , we then set the pixel value to 1 . If the pixel value falls outside of this range (less than 0.35 or greater than 0.9 ), then we set the pixel value to 0 . For some bright penumbral features whose intensity are larger than the threshold, we set values here to 1 by manual. After that, only PGs are retained, which were indicated by white contours in Figs. 1c and $\mathrm{f}$.

To label and track PGs in time, we develop a procedure with the following criteria: 1) PGs form from non-zero value pixels. Only neighboring non-zero pixels belong to the same object. Pixels forming an object are labeled by an objective identification number with which the pixel coordinates and observational times are stored in memory; 2) two objects are identified as predecessor, if they coincide in the coordinates for at least one pixel in both frames; 3 ) the lifetime is longer than $3 \mathrm{~min}$, the mean area is larger than 10 pixels or the maximum area is larger than 20 pixels; 4) it should become visible after the beginning of the observation and disappear before the ending of observation; 5) the movement of PGs in two successive images is smaller than 2 pixels, which will exclude the features that move back and forth because of the wrong tracking of poorly defined large bright features; 6) PGs that move into the umbra or surrounding granulation are also deleted in our sample, because these features are not the subject of this study. Since the south part of the penumbra is out of the FOV, we only study the subregion where the umbra and penumbra were well observed. The boundary of the subregion is indicated by white lines in Fig. 2. The procedure yielded a set of 776 PGs in the blue continuum and 413 PGs in the $\mathrm{G}$ band during the observation of $170 \mathrm{~min}$ for the blue continuum and $88 \mathrm{~min}$ for the $\mathrm{G}$ band, respectively. From the obtained outputs of pixel coordinates and observational time, we can obtain parameters, such as position, intensity, lifetime, and speed for each individual PG.

Similar to Sobotka et al. (1999) and according to the movement direction relative to the sunspot umbra, we label PGs that move toward umbra as INW and those that move toward surrounding granulation as OUT. Since the resolution of BFI is $0.2^{\prime \prime}$ and is four times the pixel size, we label PG whose movement is less than 4 pixels over the lifetime as STB. It is a new classification compared with previous studies. For the blue continuum, $421(54 \%)$ are INW, $146(19 \%)$ are OUT, and $209(27 \%)$ are STB. For the G band, $230(56 \%)$ are INW, $54(13 \%)$ are OUT, and 129 (31\%) are STB. In the following section, we describe and compare the properties of INW and OUT by first paying attention to the differences among them and then describing the properties of STB.

\section{Results}

\subsection{Properties of INW and OUT PGs}

\subsubsection{Trajectory and spatial distribution}

The trajectory of each PG is determined by a series of positions at the center of PGs. Figure 2 shows the trajectories of INW (white) and OUT (black) PGs in the blue continuum (Fig. 2a) and the G-band (Fig. 2b), respectively. The plus indicates the center of sunspot. From the figure we can see that PGs move inward in inner penumbra. In outer penumbra, most PGs move outward and few PGs move inward. Similar results can be found in Fig. 1 of Sobotka et al. (1999b). The nearest distance between the OUT PGs and the sunspot center is 9.7" $\left(9.0^{\prime \prime}\right)$ in the blue continuum (and $\mathrm{G}$ band). The mean migration distance of INW PGs is longer than that of OUT PGs. Moreover, there is no PG that passes through the whole penumbra. For the blue continuum (and G-band), the lengths of trajectories are in the range of $0.2^{\prime \prime}-3.6^{\prime \prime}\left(0.2^{\prime \prime}-2.6^{\prime \prime}\right)$ for INW PGs and $0.2^{\prime \prime}-2.3^{\prime \prime}$ $\left(0.2^{\prime \prime}-1.1^{\prime \prime}\right)$ for OUT PGs. The mean migration distance of INW and OUT PGs is $0.8^{\prime \prime}\left(0.7^{\prime \prime}\right)$ and $0.6^{\prime \prime}\left(0.5^{\prime \prime}\right)$, respectively. Figure 2 seems to indicate a much higher amount of long trajectories. However, most of them are formed by overlapping paths of PGs that appeared in the same area but at different times.

The histograms of time-averaged distances of the INW and OUT PGs from the center of umbra are shown in Fig. 3. The black dashed line represents the INW PGs, and the black solid line represents OUT PGs. The INW PGs dominate in the distance from $3^{\prime \prime}$ to $13^{\prime \prime}$ for the blue continuum (and from $3^{\prime \prime}$ to $14^{\prime \prime}$ for the G-band), i.e. in the inner penumbra, while the OUT PGs dominate in the distance from $13^{\prime \prime}$ to $16^{\prime \prime}$, i.e. in the outer penumbra. The distance $13^{\prime \prime}$ (14" for G band) from the umbral center can be considered as a dividing line between the regions of prevailing INW and OUT PGs. This position of the dividing line corresponds to $70 \%$ of the distance from the inner to the outer edge of the penumbra, similarly to what was found by Sobotka \& Sütterlin (2001). The average position of INW and OUT PGs are 10.5" and 14.7" (10.0" and 13.7" for the G-band), respectively. From the figure, we notice that the distance between the sunspot center and a few PGs are smaller than 4", which is less than the umbra radii, if we regard the umbra as a circle. The reason is that there are several bright filaments which permeated through the umbra. Since these bright filaments are very obvious, the core of these filaments are also counted as PGs.

\subsubsection{Photometric characteristics}

The brightness of each PG is obtained by averaging the maximum brightness at each time step during the whole observation. In the blue continuum (and $\mathrm{G}$ band), the brightness of PGs are in the range from $0.63(0.78)$ to 1.14 (1.12) for INW PGs 

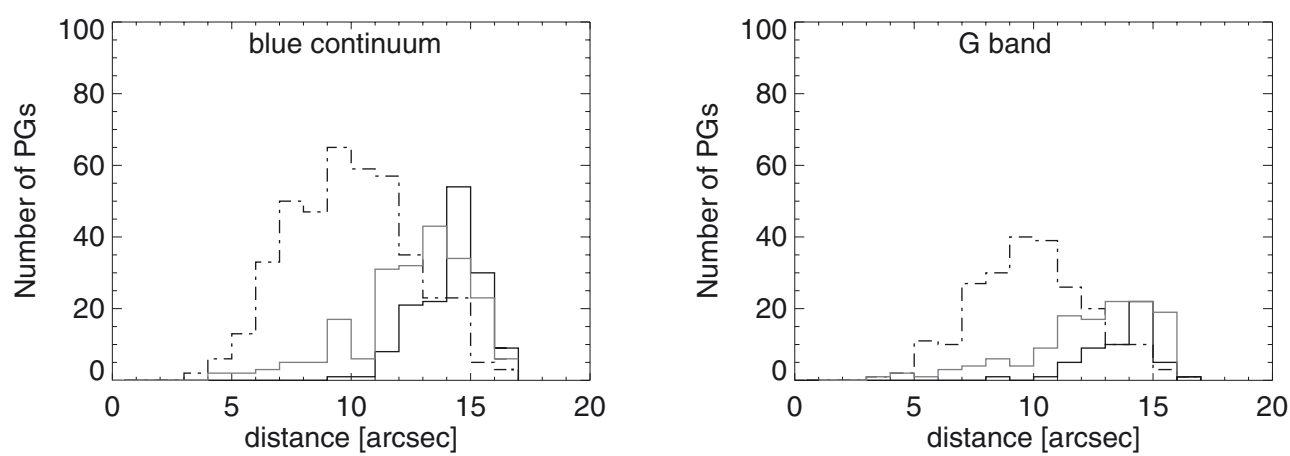

Fig. 3. Histogram of average position of PGs. The black dashed line represents INW, the block solid line represents OUT, and the gray solid line represents STB.
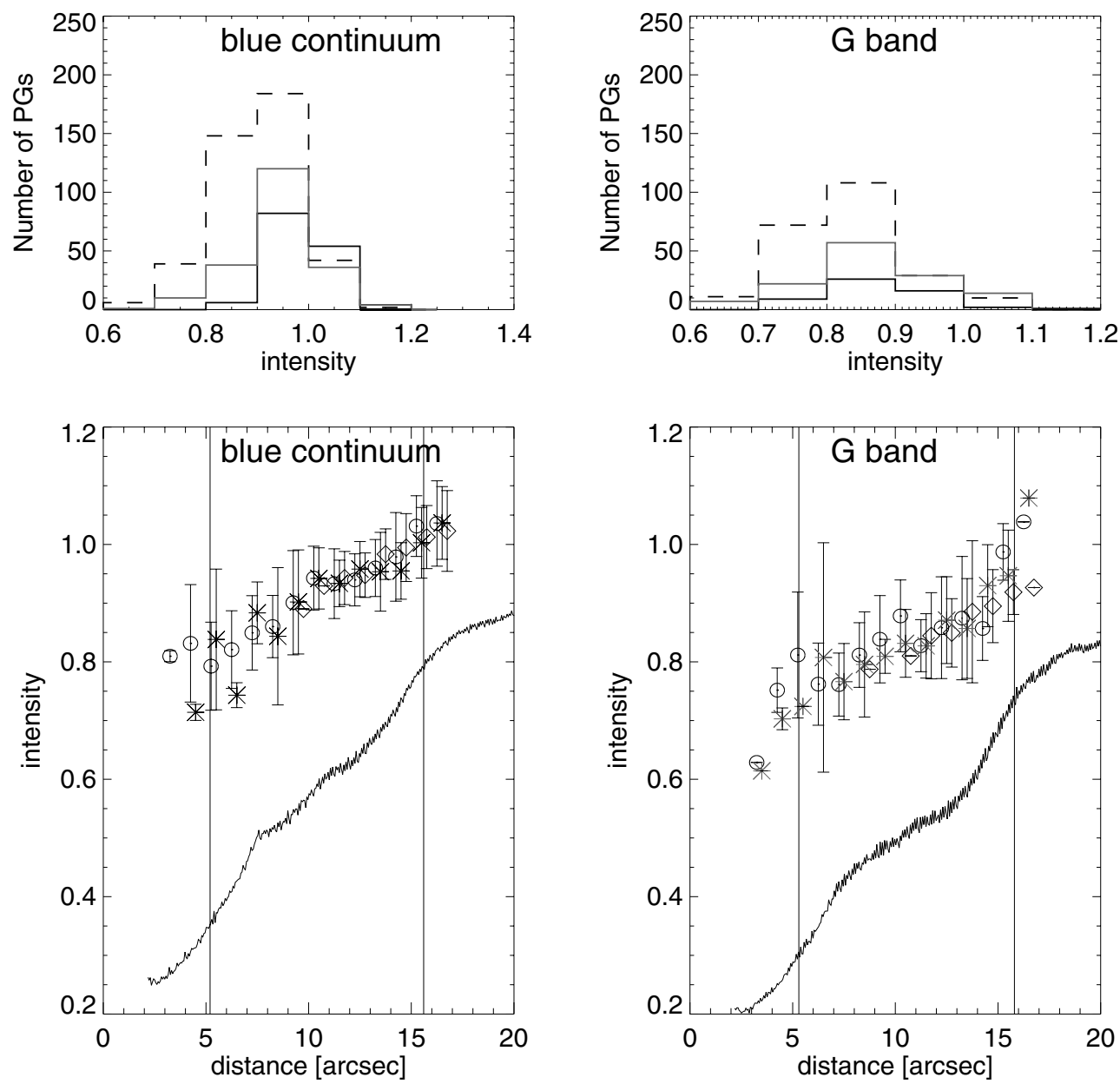

Fig. 4. Upper panels: histogram of PGs average brightness for blue continuum (left) and G band (right). Black dashed line (INW), black solid line (OUT), and gray solid line (STB). Bottom panels: average brightness as a function of position in the penumbra. Circle signs represent INW, diamond signs represent OUT, and star signs represent STB. The bars indicate a scatter of $\pm 1 \sigma$. The dark curve represents the mean intensity averaged over the azimuthal direction at each radial bin. Vertical lines represent the boundary of umbra and penumbra and the boundary of penumbra and surrounding granulation, respectively. The intensity here is measured by the intensity of PG relative to quiet Sun intensity.

and from $0.82(0.79)$ to 1.17 (1.18) for OUT PGs. Meanwhile, the average brightness for INW and OUT PGs is $0.89(0.88)$ and 0.99 (0.98) for the blue continuum (and $\mathrm{G}$ band), respectively. It is worth noting that there are often larger-scale intensity variations within the penumbra. An individual bright filament can have a lower intensity than a dark filament elsewhere. Consequently, the term PG has only local meaning and the intensity of each PG varies from one to another. The property of brightness also can be seen from histograms, as shown in the top panels in Fig. 4.

To study the dependence of brightness on position for both types of PGs, we divide the sunspot into several bins in radius, each covers 1 " from the center of the sunspot to the surrounding granulation. Brightness of all PGs whose time-averaged positions fell into a given bin were averaged. The results are shown in the bottom panels of Fig. 4. Circle signs represent INW PGs, 
Y. Zhang and K. Ichimoto: Properties of sunspot penumbral grains observed with Hinode
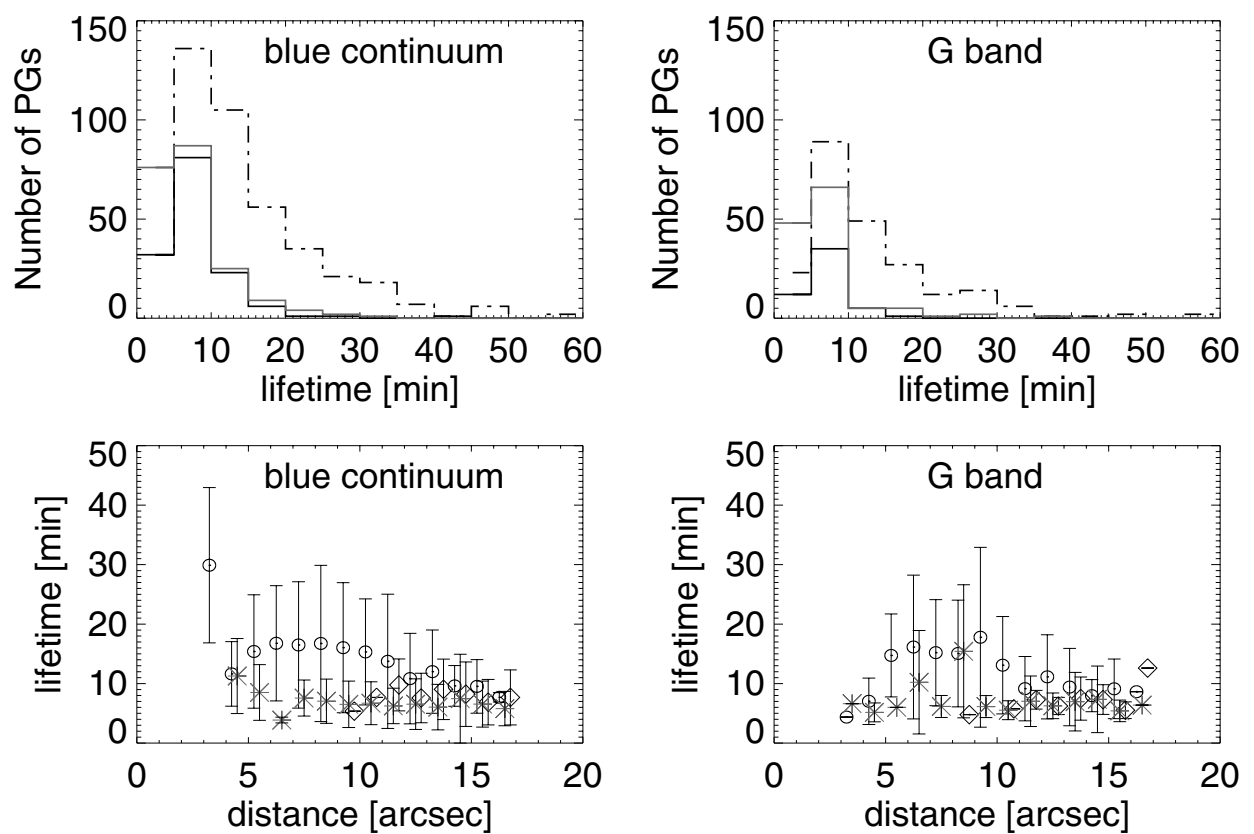

Fig. 5. Upper panels: histogram of PGs average lifetime. Black dashed line (INW), black solid line (OUT), and gray solid line (STB). Bottom panels: averaged lifetimes as a function of position in the penumbra measured by the distance from the center of the umbra. Circle signs represent INW, diamond signs represent OUT, and star signs represent STB. The bars indicate a scatter of $\pm 1 \sigma$.

and diamond signs represent OUT PGs with error bars shown the standard deviation in radial bin. The mean intensity averaged over the azimuthal direction at each radial bin is indicated by the solid curve. Two vertical lines represent the boundary between surrounding granulation and penumbra and between penumbra and umbra. The first impression of the figure shows that all symbols, such as circles, stars, and diamonds are mixed and increase as a function of the distance from the sunspot center. This indicates that the brightness of OUT PGs and INW PGs are almost the same around the same distance. Because INW PGs prevail in inner penumbra and OUT PGs prevail in outer penumbra, the average brightness value of INW PGs is naturally smaller than that of OUT PGs. It means that the difference of average brightness between both types may result from the distance from the sunspot center rather than difference types.

Looking through the evolution of each PG, we find that most PGs look like an ellipse, so we fit PGs as an ellipse to do complementary automatic measurements of lengths and widths. In doing this calculation, we use the frame on which the area of PGs reach the maximum. In the blue continuum (and $\mathrm{G}$ band), the average long axes and short axes are $0.52^{\prime \prime}\left(0.59^{\prime \prime}\right)$ and $0.23^{\prime \prime}\left(0.23^{\prime \prime}\right)$ for INW PGs and $0.57^{\prime \prime}\left(0.52^{\prime \prime}\right)$ and $0.25^{\prime \prime}$ $\left(0.23^{\prime \prime}\right)$ for OUT PGs. The eccentricity of both types are almost the same at about $0.90(0.89)$.

\subsubsection{Lifetimes}

We show histograms of the lifetime of PGs in the upper panels of Fig. 5. The lifetime here represents the time period of PG from appearance to disappearance. In our sample, two INW PGs in the blue continuum and one PG in the $G$ band lives longer than $1 \mathrm{~h}$, so we set the upper limit of $60 \mathrm{~min}$ in Fig. 5. The INW and OUT PGs show different properties of lifetime. In the blue continumm (and G band) for the INW PGs, the estimated mean lifetime is $14.7(13.5) \mathrm{min}$ and the longest lifetime is 75.3 (66.8) $\mathrm{min}$. For OUT PGs, the estimated mean lifetime is 8.0 (7.0) $\mathrm{min}$, and the longest lifetime is 41.3 (26.0) $\mathrm{min}$.
Thus, the lifetime of INW PGs tends to be longer than that of OUT PGs.

The lifetime of PGs as a function of position is shown in the bottom panels of Fig. 5. Lifetime of all PGs whose timeaveraged positions fell into a given bin were averaged. It is easy to find that the lifetime of INW PGs, which locate in inner penumbra, is longer than that of INW PGs which locate in outer penumbra. While for OUT PGs, the variation in lifetime along the position is low. Even though we only have a few OUT PGs located in the inner penumbra, it is easy to find that the lifetime of INW PGs is much longer than that of OUT PGs in the inner penumbra, while the lifetime of INW PGs and OUT PGs is almost the same in the out-penumbra. Similar results can be found in Fig. 3 in Sobotka et al. (1999b).

\subsubsection{Speed of proper motions}

The histograms of time-averaged proper motion speed are shown in the top panels of Fig. 6. For the blue continuum (and $\mathrm{G}$ band), the speed of INW PGs range from $0.13 \mathrm{~km} \mathrm{~s}^{-1}$ $\left(0.12 \mathrm{~km} \mathrm{~s}^{-1}\right)$ to $2.13 \mathrm{~km} \mathrm{~s}^{-1}\left(2.15 \mathrm{~km} \mathrm{~s}^{-1}\right)$ with a mean value around $0.71 \mathrm{~km} \mathrm{~s}^{-1}\left(0.70 \mathrm{~km} \mathrm{~s}^{-1}\right)$. There is only one INW PG, which moves faster than $2 \mathrm{~km} \mathrm{~s}^{-1}$ for both wavelengths. For OUT PGs, the speed range from $0.19 \mathrm{~km} \mathrm{~s}^{-1}\left(0.23 \mathrm{~km} \mathrm{~s}^{-1}\right)$ to $3.7 \mathrm{~km} \mathrm{~s}^{-1}\left(1.9 \mathrm{~km} \mathrm{~s}^{-1}\right)$ with a mean value around $0.93 \mathrm{~km} \mathrm{~s}^{-1}$ $\left(0.65 \mathrm{~km} \mathrm{~s}^{-1}\right)$. There are seven (one) OUT PGs moving faster than $2 \mathrm{~km} \mathrm{~s}^{-1}$ for the blue continuum (and G band). Meanwhile, the histograms show that the speed distribution of STB and INW PGs have a clear peak around 0 and $0.7 \mathrm{~km} \mathrm{~s}^{-1}$ for the blue continuum (around 0 and $0.6 \mathrm{~km} \mathrm{~s}^{-1}$ for the $\mathrm{G}$ band). For OUT PGs, they show, however, that their speed scatter in a broad range without a clear peak for both wavelengths. Moreover, the speed peak of STB and INW PGs for the blue continuum is more obvious than that for the $\mathrm{G}$ band. The time averaged speed as a function of position is shown in the bottom panels of Fig. 6. From the first impression, one can see that there is no obvious dependence 

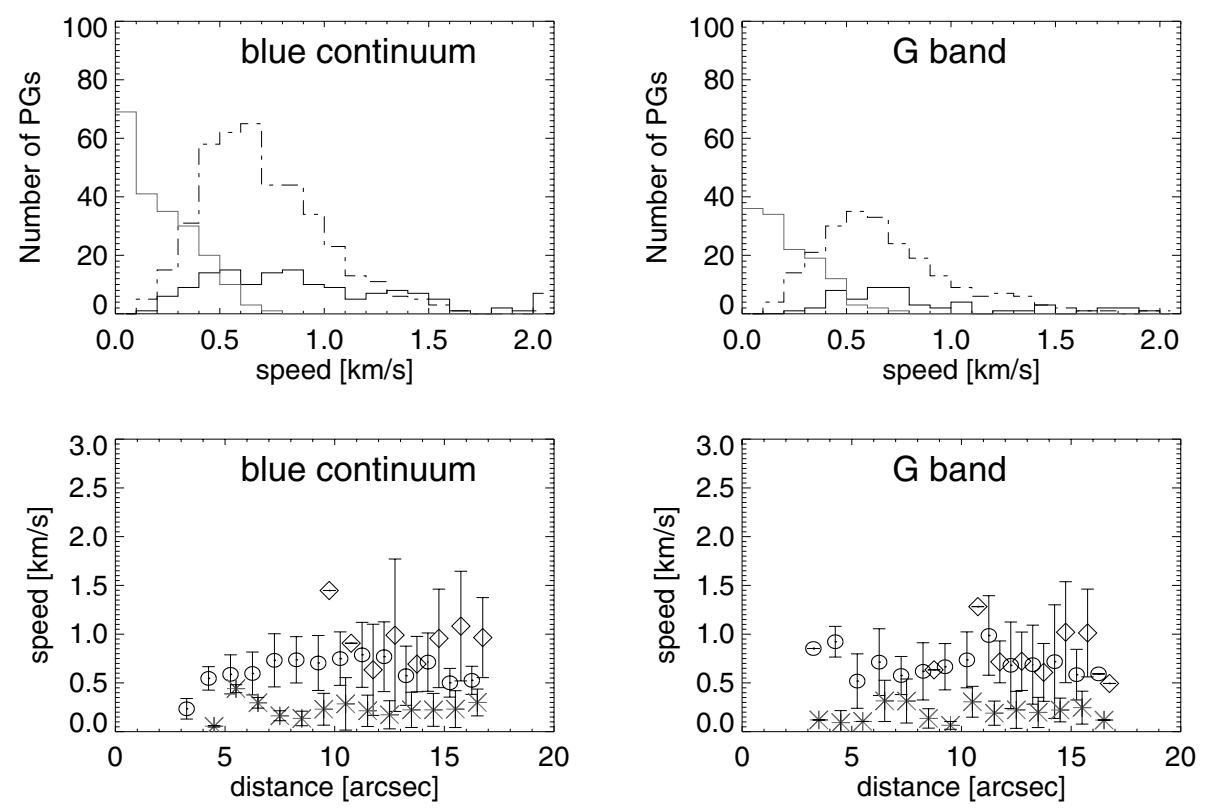

Fig. 6. Upper panels: histograms of PGs speed. Black dashed line (INW), black solid line (OUT), and gray solid line (STB). Bottom panel: speed as a function of position in the penumbra measured by the distance from the center of the umbra. Circle signs represent INW, and diamond signs represent OUT. The bars indicate a scatter of $\pm 1 \sigma$.

between the averaged speed and the position for both INW and OUT PGs. To study the dependence of speed on forming and decaying PG, we check the temporal evolution of speed for each $\mathrm{PG}$, and then find that there is no systematic difference in speed between forming phase and decaying phase.

\subsection{Properties of STB PGs}

The definition of STB PG is a PG whose trajectory is shorter than $0.2^{\prime \prime}$ with a lifetime longer than 3 min. In our statistical study, about $26 \%$ (blue continuum) and $32 \%$ (G band) of PGs belong to STB PGs. The distribution of them is from the inner penumbra, which is dominated by INW PGs, to the outer penumbra which is dominated by OUT PGs. The properties of their position, intensity, lifetime, and speed are also shown in Figs. 3-6 with gray lines and star signs. We summarize the properties of STB PGs as follows: in the blue continuum (and G band), STB PGs are located all over the penumbra with a mean position of $12.6^{\prime \prime}\left(12.4^{\prime \prime}\right)$. The intensity of STB PGs is 0.94 (0.91). The average long axes, short axes, and the eccentricity of the ellipse fit are $0.54^{\prime \prime}\left(0.51^{\prime \prime}\right), 0.21^{\prime \prime}\left(0.23^{\prime \prime}\right)$, and $0.92(0.89)$. Their mean lifetime is $7.6(7.0)$ min, which is comparable to that of OUT PGs, but significantly shorter than INW PGs.

\subsection{Temporal evolution of PGs proper motion and brightness}

The data set, which we used, have a temporal resolution of $7 \mathrm{~s}$ without a seeing effect, so it is possible for us to study the temporal evolution of the proper motion and brightness. To do the study, we construct a subsample which only contains INW and OUT PGs with lifetime longer than $10 \mathrm{~min}$. Then 326 PGs in the blue continumm and 136 PGs in the $\mathrm{G}$ band remained. For the blue continuum, $253(96 \%)$ PGs are INW, and $11(4 \%)$ are OUT. For the G band, 116 (95\%) are INW, and $6(5 \%)$ are OUT. In these long lifetime subsamples, a majority of PGs belong to INW, which is consistent with the average lifetime of INW PGs being longer than that of OUT PGs.
In our subsample, the variation in the brightness differs greatly for each PG. In the blue continuum (and G band), the maximum variation in brightness is about $0.59(0.49)$ of the quiet Sun, the minimum variation is only about $0.05(0.07)$, and the mean variation is about $0.18(0.22)$.

We show a typical evolution of brightness and speed of INW and OUT PG for the blue continuum in Fig. 7a (and G band in Fig. 7b). Stars represent the brightness and diamonds represent the speed. To easily spot the variation trends, both brightness and speed were processed by a moving average with 20 points (140 s). Meanwhile, the evolution of the first five minutes were excluded for some arbitrary motion resulting from the rapid deformation at the appearance period of PGs. From the figure, we can see that the value of brightness increases at first and then decreases, while the temporal evolution of the speed is more complex. There is no obvious tendency of the speed variation during the PG evolution.

After looking through all the temporal evolution curves, as shown in Figs. 7a and b, we find that there is no strong correlation between the brightness and the speed for the majority of PGs, though a small fraction of PGs with short lifetimes have significant positive or negative relation between brightness and speed. The correlation coefficient between brightness and speed as the function of the mean speed of each PG is shown in Fig. 7c (blue continuum) and Fig. 7d ( $\mathrm{G}$ band), where the radius of the circle is proportional to the lifetime. From these scatter plots, we see that few PGs with short lifetimes have strong positive or negative correlation between brightness and speed, while the value of the correlation coefficient is small for major PGs.

At the end of this section, we summarize the properties of PGs in Table 1.

\section{Discussion and conclusion}

By using simultaneous high spatial and temporal resolution data in the blue continuum (4504 $\AA$ ) and G-band (4305 $\AA$ ) obtained by SOT/Hinode, we study the properties of penumbral grains 
Y. Zhang and K. Ichimoto: Properties of sunspot penumbral grains observed with Hinode
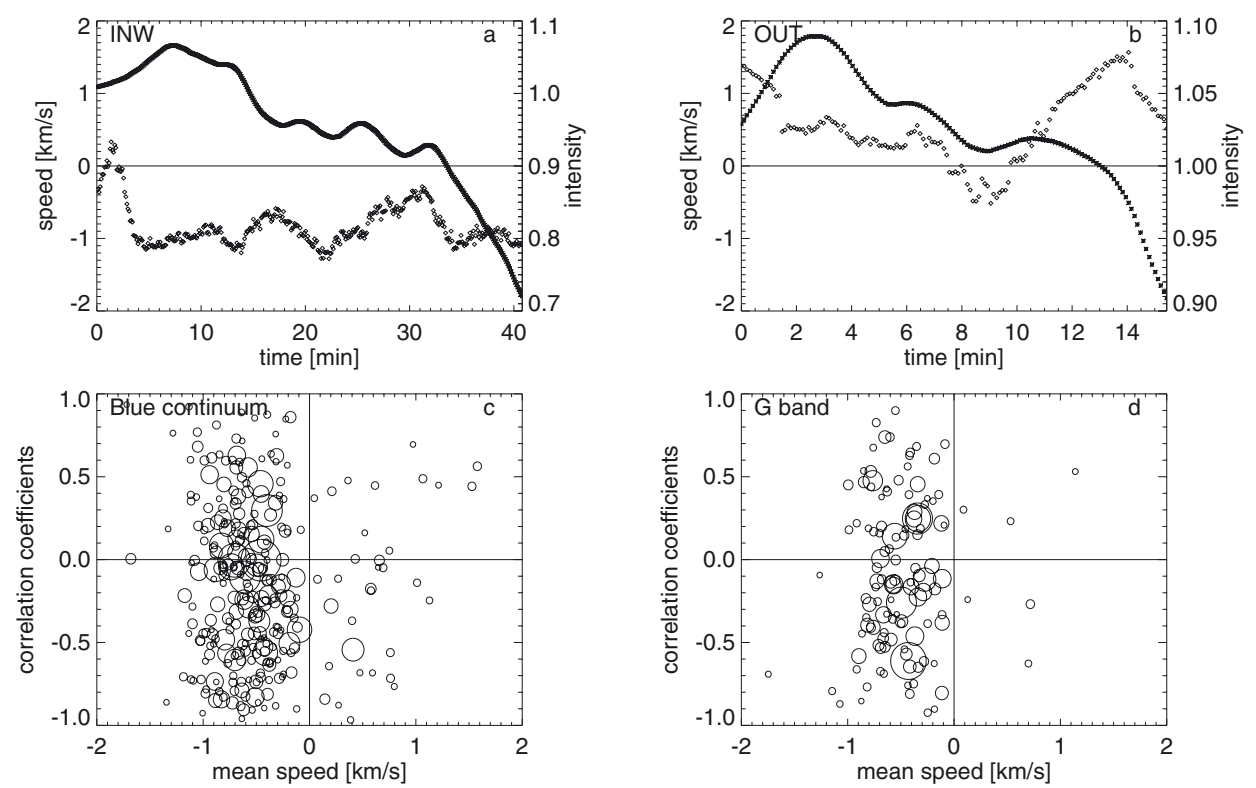

Fig. 7. Scatter plot in panels a) and b) show the temporal evolution of brightness and speed of proper motion, while the scatter plot in panels c) and d) show the correlation coefficients between brightness and speed as a function of the mean speed for blue continuum (left panel) and G band (right panel), respectively.

Table 1. Properties of penumbral grains.

\begin{tabular}{|c|c|c|c|c|c|c|c|c|c|c|c|c|}
\hline \multirow{3}{*}{ Parameters } & \multicolumn{6}{|c|}{ Blue continuum (4504 ̊̊) } & \multicolumn{6}{|c|}{ G band $(4305 \AA)$} \\
\hline & \multicolumn{2}{|c|}{ INW $(54 \%)$} & \multicolumn{2}{|c|}{ OUT $(19 \%)$} & \multicolumn{2}{|c|}{ STB $(27 \%)$} & \multicolumn{2}{|c|}{ INW $(56 \%)$} & \multicolumn{2}{|c|}{ OUT $(13 \%)$} & \multicolumn{2}{|c|}{ STB $(31 \%)$} \\
\hline & mean & $\max$ & mean & $\max$ & mean & $\max$ & mean & $\max$ & mean & $\max$ & mean & $\max$ \\
\hline Lifetime(min) & 14.7 & 75.3 & 8.0 & 41.34 & 7.6 & 31.0 & 13.5 & 66.8 & 7.0 & 16.0 & 7.0 & 35.4 \\
\hline Trajectory(") & 0.8 & 3.6 & 0.6 & 2.3 & & & 0.7 & 2.6 & 0.5 & 1.1 & & \\
\hline Position(") & 10.0 & & 14.1 & & 12.6 & & 9.9 & & 13.7 & & 12.4 & \\
\hline Speed $\left(\mathrm{km} \mathrm{s}^{-1}\right)$ & 0.71 & $2.13(2)^{a}$ & 0.93 & $3.7(7)$ & & & 0.70 & $2.15(1)$ & 0.85 & 1.9 & & \\
\hline Intensity & 0.89 & 1.14 & 0.99 & 1.17 & 0.94 & 1.14 & 0.88 & 1.12 & 0.98 & 1.18 & 0.91 & 1.19 \\
\hline Long axes $\left({ }^{\prime \prime}\right)^{b}$ & 0.52 & & 0.57 & & 0.51 & & 0.59 & & 0.52 & & 0.51 & \\
\hline Short axes $\left({ }^{\prime \prime}\right)^{b}$ & 0.23 & & 0.25 & & 0.21 & & 0.23 & & 0.23 & & 0.21 & \\
\hline Eccentricity $^{b}$ & 0.90 & & 0.90 & & 0.91 & & 0.89 & & 0.89 & & 0.91 & \\
\hline
\end{tabular}

Notes. ${ }^{(a)}$ Number of penumbral grains with speed faster than $2 \mathrm{~km} \mathrm{~s}^{-1}$; $^{(b)}$ average long axes, short axes, and eccentricity if we do an ellipse fit according the shape.

(PGs). According to the direction of movement, we classify PGs into three groups: INW PGs (move toward the umbra), OUT PGs (move toward the surrounding granulation), and STB PGs (trajectory is shorter than 4 pixels). Their properties are summarized in Table 1.

The principal statistical studies of PGs have been carried out mainly in the 1970s, when PGs were first identified, and around 2000s, when sub-arcsecond data were obtained thanks to the development of observing techniques for attain high resolution. Before comparing our results with previous ones, it is worth noting that there are several reasons, which will result in some differences: 1) the data used in the present work were obtained by a space telescope, therefore, they are free of seeingeffects. The temporal and spatial resolution is $7 \mathrm{~s}$ and $0.2^{\prime \prime}$, respectively. Both conditions ensure that we still have enough data to trace their evolution, even for the transient and small PGs. Thus we set the threshold of lifetime and area as $3 \mathrm{~min}$ and 10 pixels for an average area or 20 pixels for a maximum area. This means that some transient and small PGs will be included in our sample, which will result in some differences in the statistical properties of PGs, especially in the lifetime; 2) in identifying PGs, we use objective criterion and run an automatic procedure, which avoids the subjective effect if we do the same identification by eye; 3 ) the brightness of PGs only has local significance, which means that bright features in one part of a penumbra may be darker than the dark structures in another part. Moreover, PGs were isolated by an empirically determined threshold, which makes the identification a little subjective. Even using the same procedure, different thresholds will give different samples. Here, we use the threshold of 0.1 for blue continuum and 0.14 for $G$ band and show the PGs that were identified by the procedure in Figs. 1c and f. One can see that PGs in the present study are defined as the compact part of bright penumbral features, that only the core in the comet-like bright features are studied. Since there is no uniform criteria of the brightness of PGs, we should bear in mind that PGs identified in different studies are a little bit different. Beside all the differences mentioned above, the size of the sunspot, the evolution phase of the sunspot, the wavelength of the data, the instrument, and other details in analyzing data may also result in the difference. 
Table 2. Comparison between different studies.

\begin{tabular}{lccccccc}
\hline \hline PG type & No. & $\begin{array}{c}\lambda \\
(\AA)\end{array}$ & Fraction & $\begin{array}{c}\text { Lifetime } \\
(\mathrm{min})\end{array}$ & $\begin{array}{c}\text { Speed } \\
\left(\mathrm{km} \mathrm{s}^{-1}\right)\end{array}$ & Intensity $^{a}$ & $\begin{array}{c}\text { Trajectory } \\
\left({ }^{\prime}\right)\end{array}$ \\
\hline \multirow{3}{*}{ INW } & $1 .{ }^{b}$ & $4680 \pm 50$ & $75 \%$ & 29 & 0.4 & 0.94 & \\
& $2{ }^{c}$ & $4305 \pm 4$ & $54 \%$ & 25.2 & 0.6 & & 1.3 \\
& $3{ }^{d}$ & $4305 \pm 4$ & $81 \%$ & 13.5 & 0.7 & 0.88 & 0.7 \\
\hline \multirow{3}{*}{ OUT } & $1 .{ }^{b}$ & $4680 \pm 50$ & $25 \%$ & 22 & 0.5 & 0.96 & \\
& $2{ }^{c}$ & $4305 \pm 4$ & $46 \%$ & 18.4 & 0.8 & & 1.3 \\
& $3 .{ }^{d}$ & $4305 \pm 4$ & $19 \%$ & 7.0 & 0.65 & 0.98 & 0.50 \\
\hline
\end{tabular}

Notes. ${ }^{(a)}$ Intensity of PG relative to quiet Sun intensity; ${ }^{(b)}$ Sobotka et al. (1999b), sunspot size $~ 30^{\prime \prime}$; ${ }^{(c)}$ Sobotka et al. (2001), sunspot size $440^{\prime \prime}$; (d) present work, sunspot size $\sim 30^{\prime \prime}$.
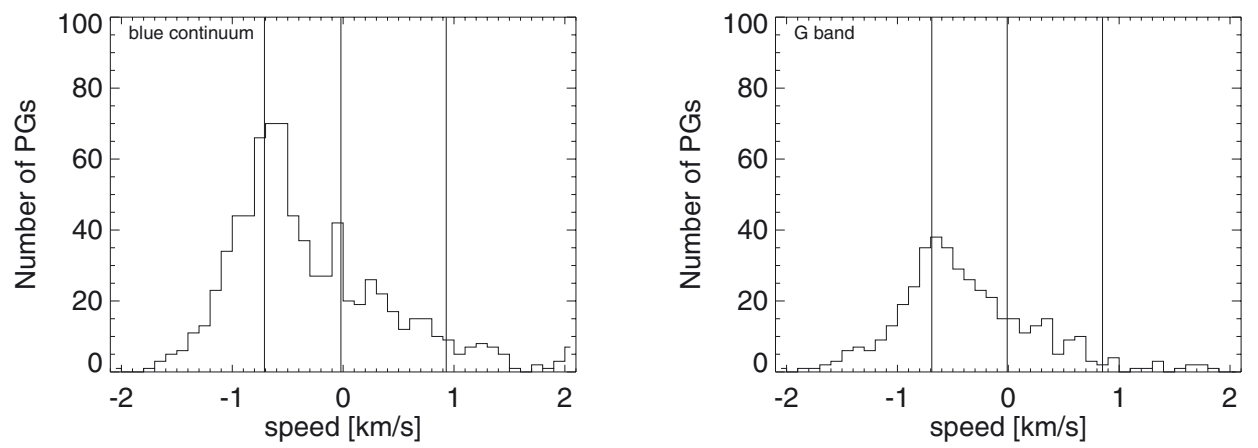

Fig. 8. Histograms of speeds for all PGs for the blue continuum (left panel) and G band (right panel). Vertical lines highlight the average speed for INW, STB, and OUT PGs.

A detailed comparison between the present and previous works are given in Table 2, which includes the sunspot size, which is estimated from the scaled-image in published papers, and the observational wavelengths. In doing this comparison, the STB PGs are neglected in the present work. The table shows that the proportion of INW and OUT PGs varies with the sunspot size. It indicates that a larger sunspot prefers to have more OUT PGs. As we motioned above, other measurement values, such as lifetime, brightness, and speed, may differ by many subjective and objective factors, so comparing the exact value of each parameter is of lesser significance. From a statistical point of view, INW PGs, however, live longer than OUT PGs, while the speed of both types has no significant difference. The brightness of difference types of PGs is similar, if they located at the same position.

Beside INW and OUT PGs, we find that about $1 / 3$ of PGs maintain static through their whole evolution. Except for maintaining static, other properties of STB PGs, such as lifetime, are similar to those of OUT PGs. A histogram of speeds for all PGs is shown in Fig. 8. In the blue continuum, there are two distinct peaks around $-0.7 \mathrm{~km} \mathrm{~s}^{-1}$ and zero, which are consistent with the average speed of INW and STB PGs. Meanwhile, there is a small peak around $0.3 \mathrm{~km} \mathrm{~s}^{-1}$. By careful examination of the upper panel of Fig. 6, we find that the number of PGs whose speed is scattered around $0.3 \mathrm{~km} \mathrm{~s}^{-1}$ is about half of the maximum for all three types. We suggest that it might result from the small peak of $0.3 \mathrm{~km} \mathrm{~s}^{-1}$. This means that the final result will be effected by the number and the speed distribution of each type of PGs when we plot the speed distribution of all PGs in one histogram. This effect can be seen more clearly in the speed histogram of all PGs in the G band. There is only one distinct peak, which consists of the average speed of INW PGs. The histograms in Fig. 8 provide some hints that the STB PGs might be a distinct class rather than the slow-moving end of the distribution of the INW and OUT classes, which deserves further simulation and theoretical studies.

In our study, there is only one OUT PG located in the inner penumbra in both wavelengths. The distance between it and the sunspot center is $9.7^{\prime \prime}\left(9.0^{\prime \prime}\right)$ in the blue continuum (and G band). Its lifetime is 4.7 (4.8) min, which is much shorter than the average lifetime of OUT PGs. It is worth noting that the sunspot is more like an ellipse with an eccentricity of 0.8 , whereas the dividing line between inner and outer penumbra is defined by a circle fit for the convenience. Thus PGs that locate the area in the vicinity to the dividing line may be misclassified. Here, we check PGs located in that area carefully and confirm that there exists one OUT PG which is located in the inner penumbra in both wavelengths. If we check the trajectory figure in Sobotka et al. $(1999,2001)$ carefully, we find that the trajectory of OUT PGs, which are located in the inner penumbra are relatively short. All studies indicate that OUT PGs do not appear within a specific distance from the umbra center. We suggest that further studies based on vector magnetograms and Doppler maps should be done to find the difference of dynamical environment for both types of PGs.

The PGs are a bright structure in the penumbra, so it is natural for us to think that they may represent localized heating within a penumbral filament. Recently, PGs are observationally identified as the location of rising hot gas that serves as the source of the Evershed flow (Ichimoto et al. 2007; Franz \& Schilchenmaier 2009). As predicted by a rising flux tube model, PGs are the foot points of the flux tube with hot gas moving upward along the tube, which will migrate from the outer edge of the penumbra towards the umbra, which can explain the migration of INW PGs in inner penumbra. What is the physical nature of OUT PGs? By summarizing the present statistical studies 
about bright features in penumbra, we can conclude that statistical properties of the INW and OUT bright features are different. However, no study yet focuses on their properties in a magnetic field and a velocity field because of the lack of observation and because the physical origin of the OUT PGs is not well understand at this point. Further observational study are needed to distinguish the difference between both types, which will then provide the observational constraints for theoretical and numerical studies.

Acknowledgements. The authors are grateful to the anonymous referee for constructive comments and detailed suggestions to this manuscript. Y. Zhang wishes to address her sincere thanks to Dr. Rob Rutten for providing the code of subsonic filter on his webpage. Y. Zhang's work is partly supported by NSFC Grant Nos. 11221063, and 11211120147, MOST Grant No. 2011CB811401, and the National Major Scientific Equipment R\&D Project ZDYZ2009-3, the Young Researcher Grant of National Astronomical Observatories, Chinese Academy of Sciences. We are grateful to the Hinode team for providing the wonderful data. Hinode is a Japanese mission developed and launched by ISAS/JAXA, with NAOJ as domestic partner and NASA and STFC (UK) as international partners. It is operated by these agencies in cooperation with ESA and NSC (Norway).

\section{References}

Deng, N., Choudhary, D. P., Tritschler, A., et al. 2007, ApJ, 671, 1013 Franz, M., \& Schilchenmaier, R. 2009, A\&A, 508, 1453

Ichimoto, K., Shine, R. A., Lites, B., et al. 2007, Publ. Astron. Soc. Japan, 59, S593

Kosugi, T., Matsuzaki, K., Sakao, T., et al. 2007, Sol. Phys., 243, 3

Min, S., \& Chae, J. 2009, Sol. Phys., 258, 203

Muller, R. 1973a, Sol. Phys., 32, 409

Muller, R. 1973b, Sol. Phys., 29, 55

Muller, R. 1976, Sol. Phys., 48, 101

Schlichenmaier, R., \& Solanki, S. K. 2003, A\&A, 411, 257

Schlichenmaier, R., Jahn, K., \& Schmidt, H. U. 1998, A\&A, 337, 897

Sobotka, M., \& Jurčák, J. 2009, ApJ, 694, 1080

Sobotka, M., \& Sütterlin, P. 2001, A\&A, 380, 714

Sobotka, M., Brand, Peter N., Simon, \& George W. 1997, A\&A, 328, 682

Sobotka, M., Brandt, P., \& Simon, G. 1999a, ASP Conf. Ser. 183, eds. T. R. Rimmele, K. S. Balasubramanian, \& R. R. Radick

Sobotka, M., Brandt, P. N., \& Simon, G. W. 1999b, A\&A, 348, 621

Solanki, S. K., \& Montavon, C. A. P. 1993, A\&A, 275, 283

Solanki, S. K., \& Rüedi, I. 2003, A\&A, 411, 249

Tönjes, K., \& Wöhl, H. 1981, Sol. Phys., 63, 69

Tsunneta, S., Suematsu, Y., Ichimoto, K., et al. 2008, Sol. Phys., 249, 167 